\title{
Global Aromaticity at the Nanoscale
}

Michel Rickhaus ${ }^{\dagger}$, Michael Jirasek ${ }^{\dagger}$, Lara Tejerina, Henrik Gotfredsen, Martin D. Peeks, Renée Haver, Hua-Wei Jiang, Timothy D. W. Claridge, and Harry L. Anderson*

University of Oxford, Department of Chemistry, Chemistry Research Laboratory, Oxford OX1 3TA United Kingdom

†These authors contributed equally

*Correspondence to: harry.anderson@chem.ox.ac.uk.

Aromaticity is an important concept for predicting electronic delocalisation in molecules, particularly for designing organic semiconductors and single-molecule electronic devices ${ }^{1}$. It is most simply defined by the ability of a cyclic molecule to sustain

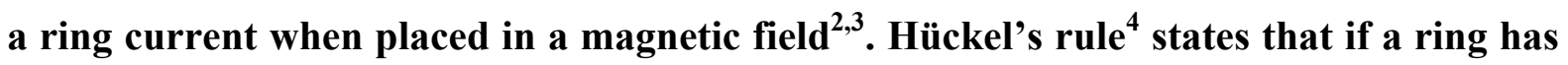
$[4 n+2] \pi$-electrons, it will be aromatic with an induced magnetisation that opposes the external field inside the ring, whereas if it has $4 n \pi$-electrons, it will be antiaromatic with the opposite magnetisation. This rule reliably predicts the behaviour of small molecules, typically with circuits of less than about $22 \pi$-electrons $(n=5)^{5}$. It is not clear whether aromaticity has a size limit and whether Hückel's rule is valid in much larger macrocycles. Here, we present evidence for global aromaticity in a wide variety of porphyrin nanorings, with circuits of up to $162 \pi$-electrons $(n=40$; diameter $5 \mathrm{~nm})$. We show that aromaticity can be controlled by changing the molecular structure, oxidation state and three-dimensional conformation. Whenever a global ring current is observed, its direction is correctly predicted by Hückel's rule. The magnitude of the current is maximised when the average oxidation state of the porphyrin units is around $0.5-0.7$, when the system starts to resemble a conductor with a partially filled valence band. Our results show that aromaticity can arise in large macrocycles, bridging the size gap between ring currents in molecular and mesoscopic rings ${ }^{6}$.

The extent of electronic delocalisation in linear molecules is limited by the onset of symmetry-breaking transitions, which can be viewed as Peierls-type electron-vibration interactions or as shifts in mixed-valence behaviour ${ }^{7}$. For example, cyanine dyes feature a 
linear chain of C-C bonds with bond order 1.5 and negligible bond length alternation (like the $\mathrm{C}-\mathrm{C}$ bonds in benzene) resulting in charge delocalisation, but if the chain exceeds a critical length, the symmetry collapses, localising the charge $e^{8,9}$. It is not clear whether similar effects limit the size of an aromatic ring, or whether molecular ring currents can extend into the domain of mesoscopic phenomena such as Aharonov-Bohm oscillations ${ }^{6}$. Many new globally aromatic macrocycles have been reported during the last few years ${ }^{10-20}$, but none with more than $80 \pi$-electrons. Here we explore circuits of up to $162 \pi$-electrons in a large family of nanorings in a wide range of oxidation states. In these nanorings, each porphyrin contributes 10 electrons to the Hückel $\pi$-electron count, and each linking alkyne contributes 2 electrons, so a nanoring cation $\boldsymbol{c}-\mathbf{P} N\left[\mathbf{b}_{x} \mathbf{e}_{y}\right]^{Q+}$ has an electron count of $10 N+4 x+2 y-Q$ (where $N$ is the number of porphyrin units; $x$ and $y$ are the number of butadiyne and ethyne links respectively).

The six-porphyrin nanoring complexes $c$-P6 $\left[\mathbf{e}_{6}\right] \cdot \mathbf{T 6}^{*}, c-\mathbf{P 6}\left[\mathbf{b e}_{5}\right] \cdot \mathbf{T} \mathbf{6}^{*}, c-\mathbf{P 6}\left[\mathbf{b}_{5} \mathbf{e}\right] \cdot \mathbf{T 6}$ and $\boldsymbol{c}-\mathbf{P 6}\left[\mathbf{b}_{6}\right] \cdot \mathbf{T 6}$ provide a homologous series of compounds in which we systematically vary the number of $\pi$-electrons by changing the number of $-\mathrm{C} \equiv \mathrm{C}$ - units, while preserving the circular geometry (which is locked by the template, T6* or T6, Figure 1 ) ${ }^{21,22}$. The ${ }^{1} \mathrm{H}$ NMR spectra of the $2+, 4+$ and $6+$ oxidation states of all four complexes reveal the presence of aromatic or antiaromatic ring currents, and the directions of these ring currents agree perfectly with Hückel's rule. Thus, $\boldsymbol{c}-\mathbf{P 6}\left[\mathbf{e}_{\mathbf{6}}\right] \cdot \mathbf{T} \mathbf{6} *$ (neutral: $72 \pi \mathrm{e}$ ) and $\boldsymbol{c}-\mathbf{P 6}\left[\mathbf{b}_{\mathbf{6}}\right] \cdot \mathbf{T} \mathbf{6}$ (neutral: $84 \pi \mathrm{e}$ ) are both aromatic in the $2+$ and $6+$ oxidation states and antiaromatic in the

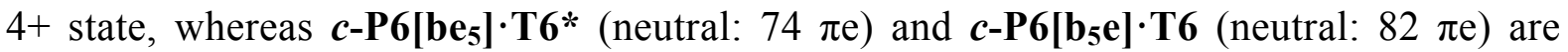
both antiaromatic in the $2+$ and $6+$ states and aromatic in the $4+$ state. The most obvious evidence for these ring currents comes from the chemical shifts of the template $\alpha$ and $\beta$ pyridyl ${ }^{1} \mathrm{H}$ resonances; for example, these protons are strongly shielded $\left(\delta_{\mathrm{H}}=-7.3\right.$ and $3.6 \mathrm{ppm}, v s .8 .7$ and 7.5 , respectively in the free template) in aromatic $\boldsymbol{c}-\mathbf{P 6}\left[\mathbf{e}_{\mathbf{6}}\right] \cdot \mathbf{T 6}^{*{ }^{2+}}$ and strongly deshielded $\left(\delta_{\mathrm{H}}=36.0\right.$ and $30.1 \mathrm{ppm}$, respectively) in antiaromatic $\boldsymbol{c}$-P6 $\left[\mathbf{e}_{\mathbf{6}}\right] \cdot \mathbf{T} \mathbf{6}^{* *^{4+}}$. Further evidence is provided by the trihexylsilyl (THS) ${ }^{1} \mathrm{H}$ and ${ }^{13} \mathrm{C}$ signals. In each spectrum, we observe one group of THS signals that is essentially unshifted, at $0-2 \mathrm{ppm}$ as in the neutral compounds THS $_{\text {out, }}$, coloured orange in Figure 1, near the zero-shielding cone of the nanoring), and one group of THS signals that is shielded or deshielded, depending on the direction of the ring current $\left(\mathrm{THS}_{\mathrm{in}}\right.$, coloured green in Figure 1). Interconversion of $\mathrm{THS}_{\text {in }}$ and $\mathrm{THS}_{\text {out }}$ is slow on the NMR timescale and the assignment of THS $_{\text {in }}$ signals is confirmed by the observation of NOEs to protons of the template (T6 or 
a)
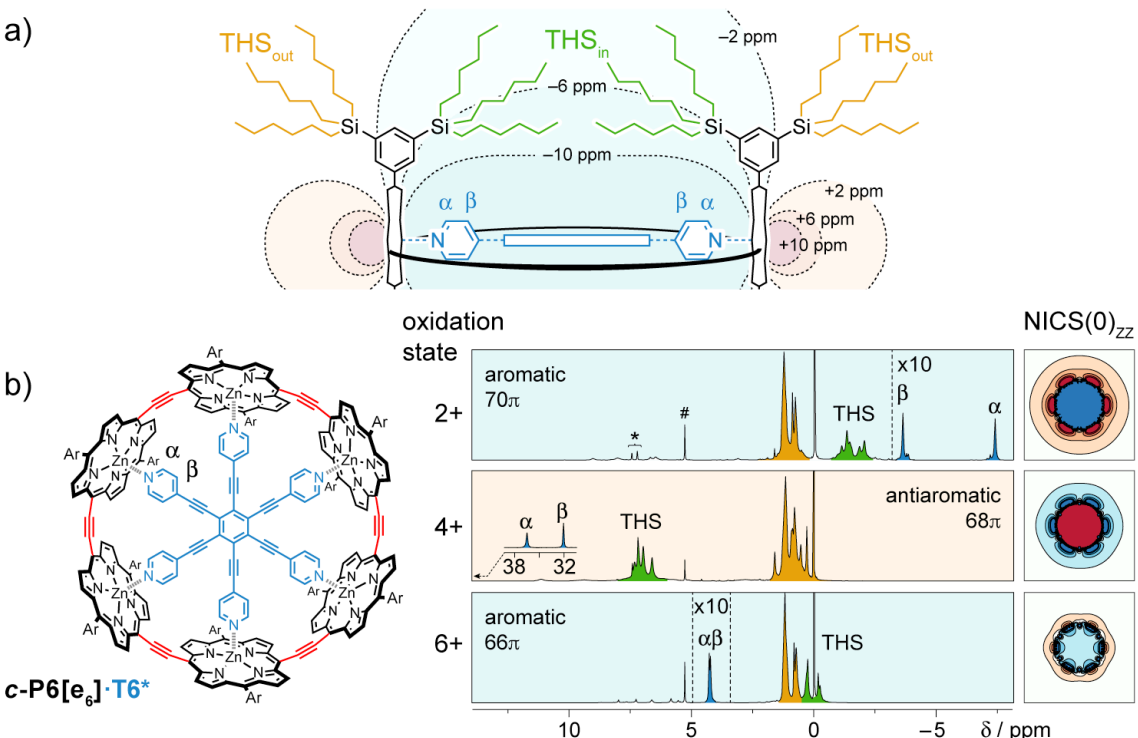

c)
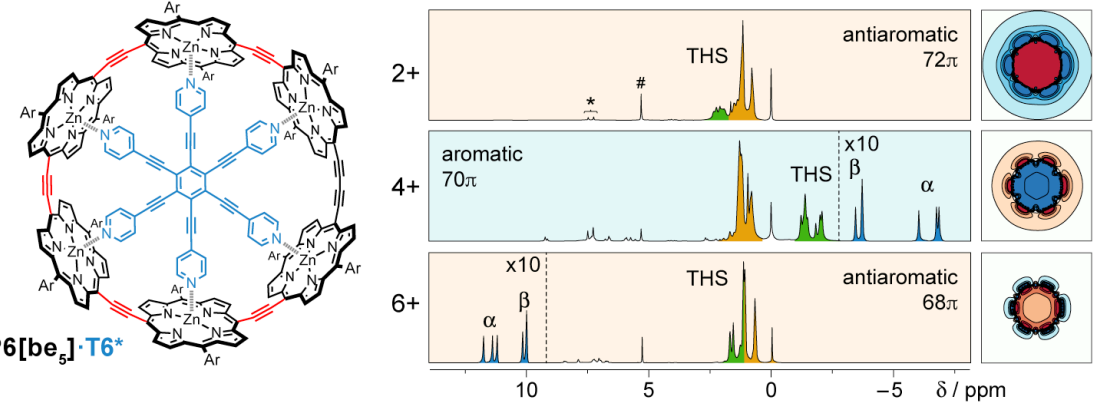

d)
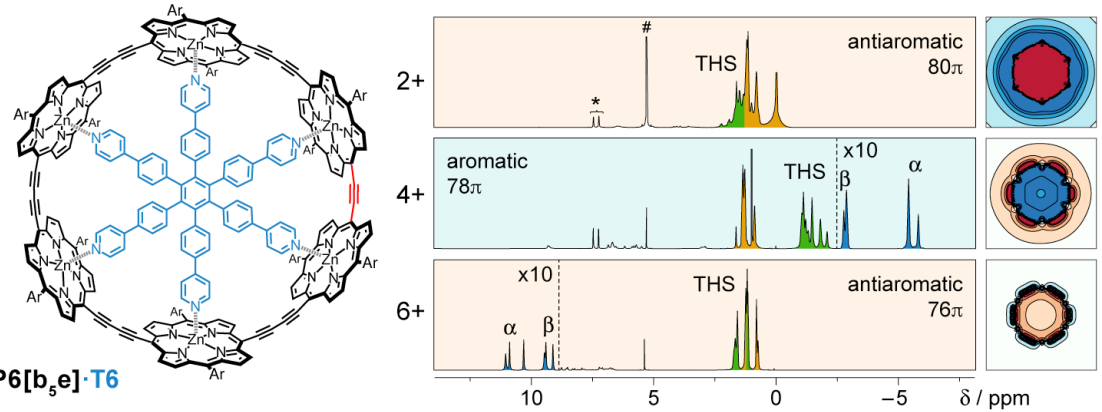

e)
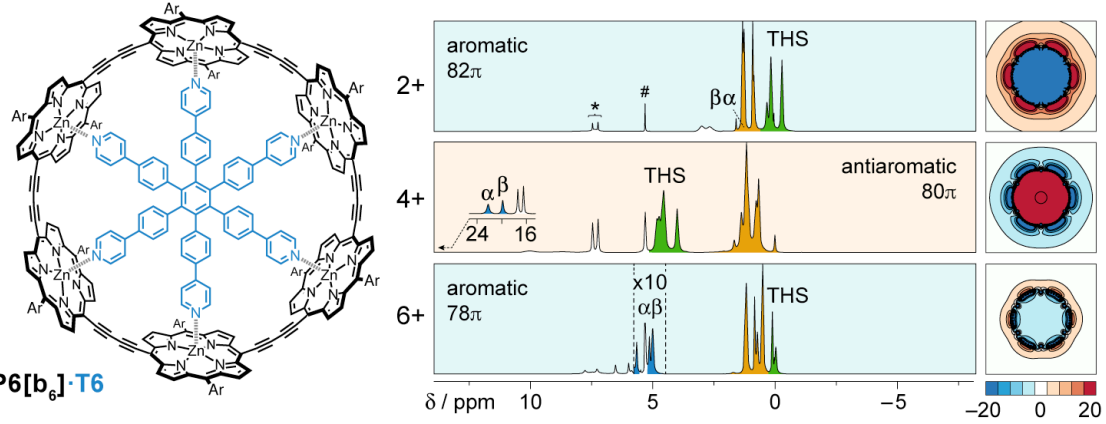

Figure 1. Aromatic and antiaromatic states of a family of oxidised six-porphyrin nanorings.

a) Side view of a nanoring. Internal trihexylsilyl groups $\left(\mathrm{THS}_{\mathrm{in}}\right)$ are sensitive to the global ring current whereas external ones $\left(\mathrm{THS}_{\text {out }}\right)$ are not. $\mathrm{NICS}(x z)_{\text {iso }}$ contours are drawn for $\left(\boldsymbol{c}-\mathbf{P 6}\left[\mathbf{e}_{\mathbf{6}}\right] \cdot \mathbf{T} \mathbf{6}^{*}\right)^{\mathbf{2}+}$ from -10 to $10 \mathrm{ppm} .{ }^{1} \mathrm{H}$ NMR spectra of the template complexes: (b) $c$-P6 $\left[\mathbf{e}_{6}\right] \cdot \mathbf{T 6 *}$, (c) $c$-P6 $\left[\mathbf{b e} \mathbf{e}_{5}\right] \cdot \mathbf{T 6 *}$, (d) $\boldsymbol{c}-\mathbf{P 6}\left[\mathbf{b}_{5} \mathbf{e}\right] \cdot \mathbf{T 6}$ and (e) $c$ $\mathbf{P 6}\left[\mathbf{b}_{6}\right] \cdot \mathbf{T} 6$ in oxidation states $2+, 4+$ and $6+$; and corresponding $\mathrm{NICS}(0)_{\mathrm{zz}}$ grids in the $x-y$ plane of the nanoring without template (LC- $\omega \mathrm{HPBE} / 6-31 \mathrm{G}^{*}, \omega=0.1$; colour axis is truncated above 20 and below -20 ppm; contours are drawn every $5 \mathrm{ppm},-40$ to $40 \mathrm{ppm} ; 5 \times 5 \mathrm{~nm}$ ). ${ }^{1} \mathrm{H} \mathrm{NMR}$ spectra recorded at $500 \mathrm{MHz}$ in $\mathrm{CD}_{2} \mathrm{Cl}_{2} ;$ oxidised states are generated by titration with thianthrenium hexafluoroantimonate. \# and * denote $\mathrm{CHDCl}_{2}$ and thianthrene, respectively. Detailed spectra for each state are shown in Supplementary Figures S9-S12 and S19S34. Dashed vertical lines indicate 10-fold magnifications. 
b)

a)
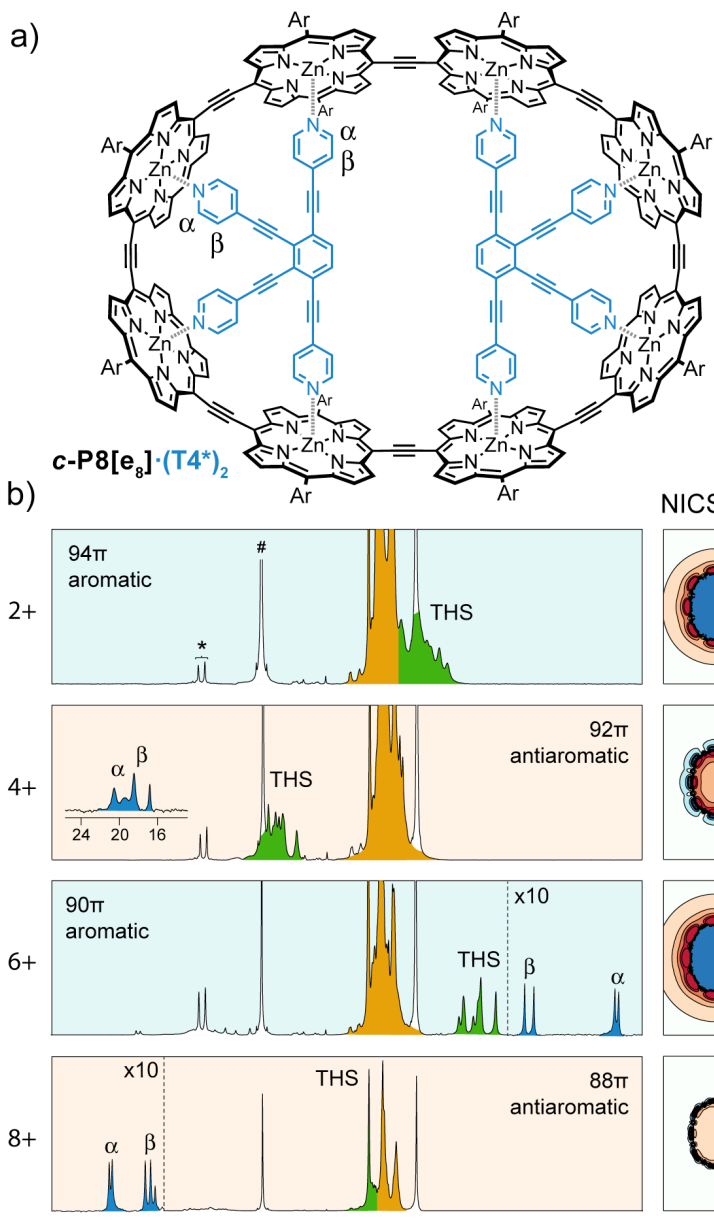

10
$\operatorname{NICS}(0)$
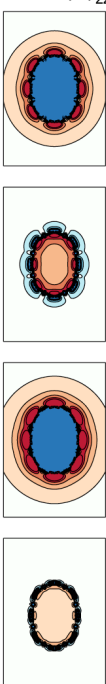

$-5 \mathrm{ppm} 20 \quad 0 \quad-20$
Figure 2. Hückel behaviour in a template-bound eight-porphyrin ring.

a) Molecular structure of $c-P 8\left[\mathbf{e}_{8}\right] \cdot(\mathbf{T} 4 *)_{2}$. Ar $=$ 3,5-bis(trihexylsilyl))phenyl. b) ${ }^{1} \mathrm{H}$ NMR spectra of oxidised $c-\mathbf{P 8}\left[\mathbf{e}_{\mathbf{8}}\right] \cdot(\mathbf{T} 4 *)_{2}$. Labels denote the most important resonances $\mathrm{THS}_{\text {in }}$ (green), $\mathrm{THS}_{\text {out }}$ (orange), and template $(\alpha, \beta)$. \# and $*$ denote $\mathrm{CHDCl}_{2}$ and thianthrene, respectively. Detailed spectra for each state are shown in Supplementary Figures S14 and S45-S47. NICS $(0)_{z z}$ grids (LC- $\omega \mathrm{HPBE} / 6-31 \mathrm{G}^{*}, \omega=$ 0.1 ) in the $x-y$ plane for each state (without template). 
Fluorinated templates allow the aromaticity of nanorings to be evaluated using ${ }^{19} \mathrm{~F}$ NMR, as exemplified by the extended six-legged template T6ef (Figure 3). Two molecules

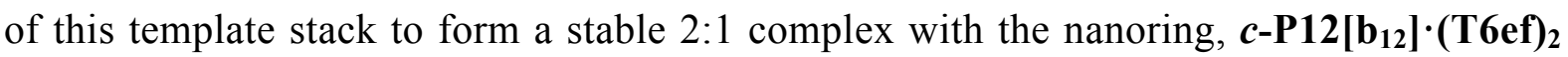
(circuit electron count: $168 \pi$ e when neutral ${ }^{23}$ ), in which the $\mathrm{CF}_{3}$ groups are positioned to probe the global ring current. The $\mathrm{CF}_{3}{ }^{19} \mathrm{~F}$ resonance is shielded in the aromatic $6+$ and $10+$ oxidation states, but deshielded in the antiaromatic $8+$ oxidation state (Figure $3 a$ ). These shifts of the $\mathrm{CF}_{3}{ }^{19} \mathrm{~F}$ resonance are fully consistent with shifts of the $\mathrm{THS}_{\text {in }}{ }^{1} \mathrm{H}$ signals (shaded green, Figure 3a) and they agree with the predictions of NICS calculations (see Supplementary Figures S87-S89). No aromatic or antiaromatic ring current was detected for the $12+$ oxidation state, and this result is reproduced by the NICS calculations, although this $12+$ state is expected to be antiaromatic $(156 \pi \mathrm{e} ; 4 n ; n=39)$. The ${ }^{19} \mathrm{~F}$ NMR titrations also show $\mathrm{CF}_{3}$ signals attributed to the open-shell $7+, 9+$ and $11+$ oxidation states, at similar chemical shifts to the neutral compound - thus the shielding or deshielding effects in the open-shell cations are small compared with those in closed-shell species. EXSY NMR experiments show that the odd-electron oxidation states are in chemical exchange with the even-electron states, on a timescale of seconds. It is not surprising that mixtures of oxidation states are formed during these titrations, because the oxidation potentials are closely spaced (for calculated speciation curves, see Supplementary Figure S79), but it is remarkable that these open-shell species give spectra that are sharp enough to be observed.

We used the 12-porphyrin nanoring $\mathbf{c}-\mathbf{P 1 2}\left[\mathbf{b}_{12}\right]$ to explore the relationship between 3D conformation and aromaticity. The magnitude of the ring current induced in a macroscopic ring of metal wire depends on the total magnetic flux passing through the ring. If the ring has a figure-of-eight shape, with two equal lobes such that the magnetic flux passing through each loop induces equal and opposite currents, then there will be no net ring current. We sought to test whether this principle applies on the molecular scale, so we synthesised a small six-legged template, T6f, which forms a figure-of-eight shaped ${ }^{23}$ 1:2 complex $\boldsymbol{c}-\mathbf{P 1 2}\left[\mathbf{b}_{\mathbf{1 2}}\right] \cdot(\mathbf{T 6 f})_{\mathbf{2}}$ (note that this system is doubly twisted, not Möbius ${ }^{24,25}$, Figure $3 \mathrm{~b}$ ) and investigated the ring currents in this system by ${ }^{1} \mathrm{H}$ and ${ }^{19} \mathrm{~F}$ NMR as a function of oxidation state, under identical conditions to those used for the circular $c$ P12 [ $\left.\mathbf{b}_{12}\right] \cdot$ (T6ef) 2. The resulting ${ }^{1} \mathrm{H},{ }^{13} \mathrm{C}$ and ${ }^{19} \mathrm{~F}$ NMR spectra show the absence of any detectable ring currents $(\Delta \delta<0.1 \mathrm{ppm})$ in the figure-of-eight nanoring, confirming that aromaticity can be switched on/off by geometry. This result is reproduced by the 

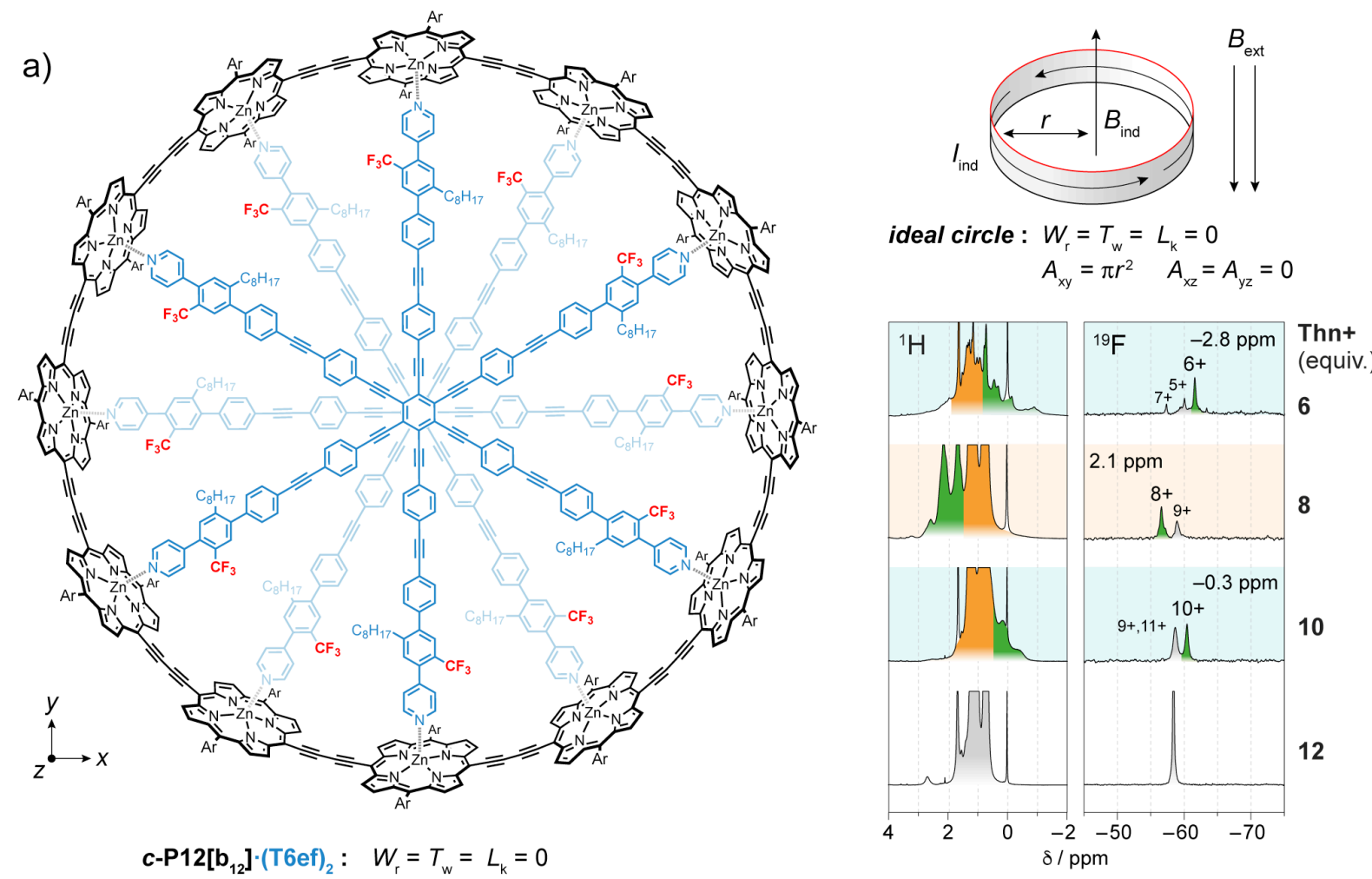

b)

$$
\begin{aligned}
c-P 12\left[b_{12}\right]^{\cdot}(\text { T6ef })_{2}: & W_{r}=T_{w}=L_{k}=0 \\
& A_{x y}=22 \mathrm{~nm}^{2} \quad A_{x z}=A_{y z}=0
\end{aligned}
$$
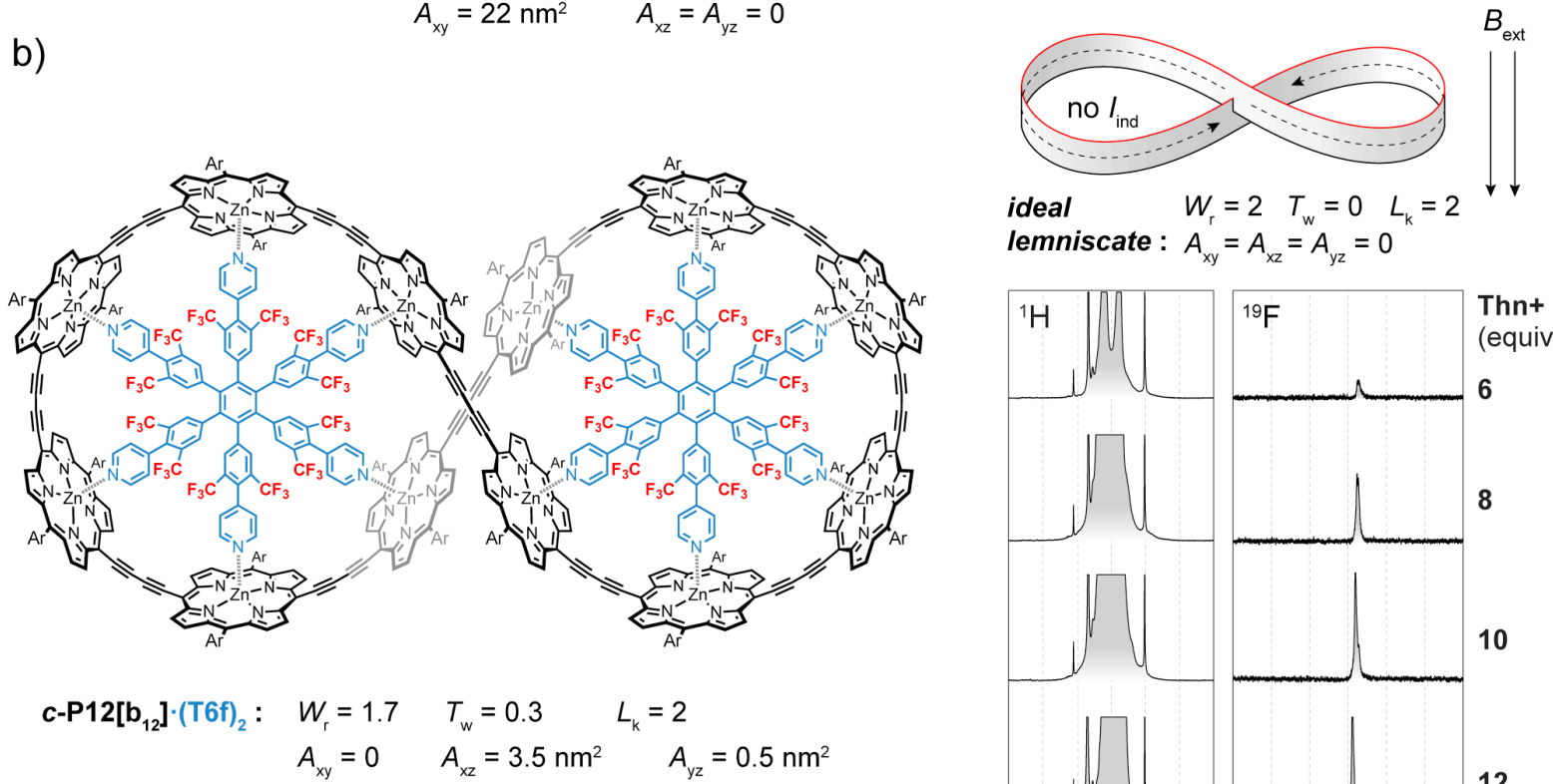

Figure 3. Ring currents in topologically distinct twelve-porphyrin ring complexes.

Left: Schematic representation of two $c-\mathbf{P 1 2}\left[\mathbf{b}_{12}\right]$ complexes forming either a circle $(\mathrm{a}): \boldsymbol{c}-\mathbf{P} 12\left[\mathbf{b}_{12}\right] \cdot(\mathbf{T} 6 \mathbf{e f})_{2}$, or a lemniscate (b): $\boldsymbol{c}-\mathbf{P 1 2}\left[\mathbf{b}_{\mathbf{1 2}}\right] \cdot(\mathbf{T 6 f})_{2}$. Both templates feature fluorine probes. Right: A circular conformation is predicted to exhibit ring currents and thus an induced magnetic moment upon the application of an external magnetic field. In the lemniscate, the two loops induce opposite currents which cancel, and no ring current is expected. ${ }^{1} \mathrm{H}$ and ${ }^{19} \mathrm{~F}-\mathrm{NMR}$ spectra of oxidised $\boldsymbol{c}-\mathbf{P} 12\left[\mathbf{b}_{12}\right] \cdot(\mathbf{T 6 e f})_{2}$ and $\boldsymbol{c}-\mathbf{P 1 2}\left[\mathbf{b}_{12}\right] \cdot(\mathbf{T 6 f})_{2}$. Green shading indicates the interior $\mathrm{THS}_{\mathrm{in}}$ resonance. Backgrounds indicate a global aromatic (blue) or antiaromatic (orange) state. Detailed spectra for each state are shown in Supplementary Figures S17, S18 and S58-S67. $L_{\mathrm{k}}, W_{\mathrm{r}}$ and $T_{\mathrm{w}}$ are the linking number, writhe and twist that specify the topology of the loop ${ }^{28} . A_{\mathrm{xy}}, A_{\mathrm{xz}}$ and $A_{\mathrm{yz}}$ are the net cross section areas; $A_{\mathrm{xy}}=0$ for $\boldsymbol{c}-\mathbf{P 1 2}\left[\mathbf{b}_{12}\right] \cdot(\mathbf{T} 6 \mathbf{f})_{2}$ because it has $D_{2}$ symmetry and the areas of the two loops cancel. 
NICS calculations (Supplementary Figure S90). The suppression of ring currents in figureof-eight shaped annulenes has been predicted theoretically ${ }^{24,26}$, but it has not been observed experimentally in other figure-of-eight shaped aromatic systems ${ }^{10,11,18,24,25,27}$, probably because they were not the right shape to achieve cancellation of the ring current. The topology of a closed ribbon can be described by the linking number $L_{\mathrm{k}}$, the writhe $W_{\mathrm{r}}$ and the twist $T_{\mathrm{w}}$ (ref. 28). Total cancellation of the ring current is expected for a geometry with $W_{\mathrm{r}}=2, T_{\mathrm{w}}=0$ and $L_{\mathrm{k}}=2$ with $D_{2}$ symmetry $^{26}$, which is close to the geometry of $c$ $\mathbf{P 1 2}\left[\mathbf{b}_{12}\right] \cdot(\mathbf{T 6 f})_{2}\left(W_{\mathrm{r}}=1.7, T_{\mathrm{w}}=0.3\right.$ and $L_{\mathrm{k}}=2$, from the crystal structure of $\boldsymbol{c}$ P12[ $\left.\mathbf{b}_{12}\right] \cdot(\mathbf{T 6})_{2}$, ref: 29). The $\boldsymbol{c}-\mathbf{P 1 2}\left[\mathbf{b}_{12}\right] \cdot(\mathbf{T 6 e f})_{2}$ ring has a large cross sectional area $\left(A_{\mathrm{xy}}=\right.$ $22 \mathrm{~nm}^{2} ; A_{\mathrm{xz}}=A_{\mathrm{yz}}=0$; Figure $3 \mathrm{a}$ ) resulting in a substantial ring current, whereas the $c$ $\mathbf{P 1 2}\left[\mathbf{b}_{12}\right] \cdot(\mathbf{T 6 f})_{2}$ lemniscate has a small net cross section $\left(A_{\mathrm{xy}}=0, A_{\mathrm{xz}}=3.5 \mathrm{~nm}^{2}, A_{\mathrm{yz}}=\right.$ $0.5 \mathrm{~nm}^{2}$; Figure $3 \mathrm{~b}$ and Supplementary Figure S92) resulting in a weak response to magnetic field. The ring current in $\boldsymbol{c}-\mathbf{P 1 2}\left[\mathbf{b}_{12}\right] \cdot(\mathbf{T 6 f})_{2}$ is blocked by the global topology $\left(T_{\mathrm{w}}\right.$ $\approx 0$ and $W_{\mathrm{r}} \approx 2$ ), not by any local break in $\pi$-conjugation.

The ring currents observed in this whole family of nanorings are summarised in Figure 4 , which plots the shift in the THS in resonances $\left(\Delta \delta_{\mathrm{THSin}}\right)$ as a function of oxidation state. Whenever a ring current is observed, its direction (aromatic or antiaromatic) matches the prediction from Hückel's rule. The magnitude of the ring current varies with the average oxidation state of the porphyrin units $\left(\overline{P_{\mathrm{OX}}}=Q / N\right)$. The largest ring currents are observed in mixed-valence systems, where $\overline{P_{\mathrm{OX}}} \approx+0.5$ to +0.7 . Global ring currents are not observed in the neutral rings $\left(\overline{P_{\mathrm{OX}}}=0\right)$, where the local porphyrin ring current dominates; in the larger rings, the ring current also vanishes when $\overline{P_{\mathrm{OX}}}=1$ (see square points for $\boldsymbol{c}-\mathbf{P 8}\left[\mathbf{b}_{\mathbf{8}}\right] \cdot(\mathbf{T} 4)_{2}{ }^{\mathbf{8}^{+}}, \boldsymbol{c}$ $\mathbf{P 1 0}\left[\mathbf{b}_{10}\right] \cdot(\mathbf{T 5})_{2}{ }^{10+}$ and $c-P 12\left[\mathbf{b}_{12}\right] \cdot(\text { T6ef })_{2}{ }^{12+}$ in Figure 4). The formation of a mixed valence state appears to be essential for efficient nanoscale charge delocalisation, just as the presence of a partially filled band is essential for conductance in an extended lattice ${ }^{30}$.

Hückel's rule was originally formulated to explain the unusual properties of benzene, and other molecules with $6 \pi$-electrons ${ }^{4}$. It is remarkable that this simple rule correctly predicts the magnetic response of large oxidised nanorings with circuits of up to $162 \pi$ electrons. This work shows that electronic delocalisation can extend coherently around molecular rings with circumferences of $16 \mathrm{~nm}$. These supramolecular rings allow the magnitude of the ring current to be controlled by topology and by the oxidation state in a way that has not yet been demonstrated for small molecules. 

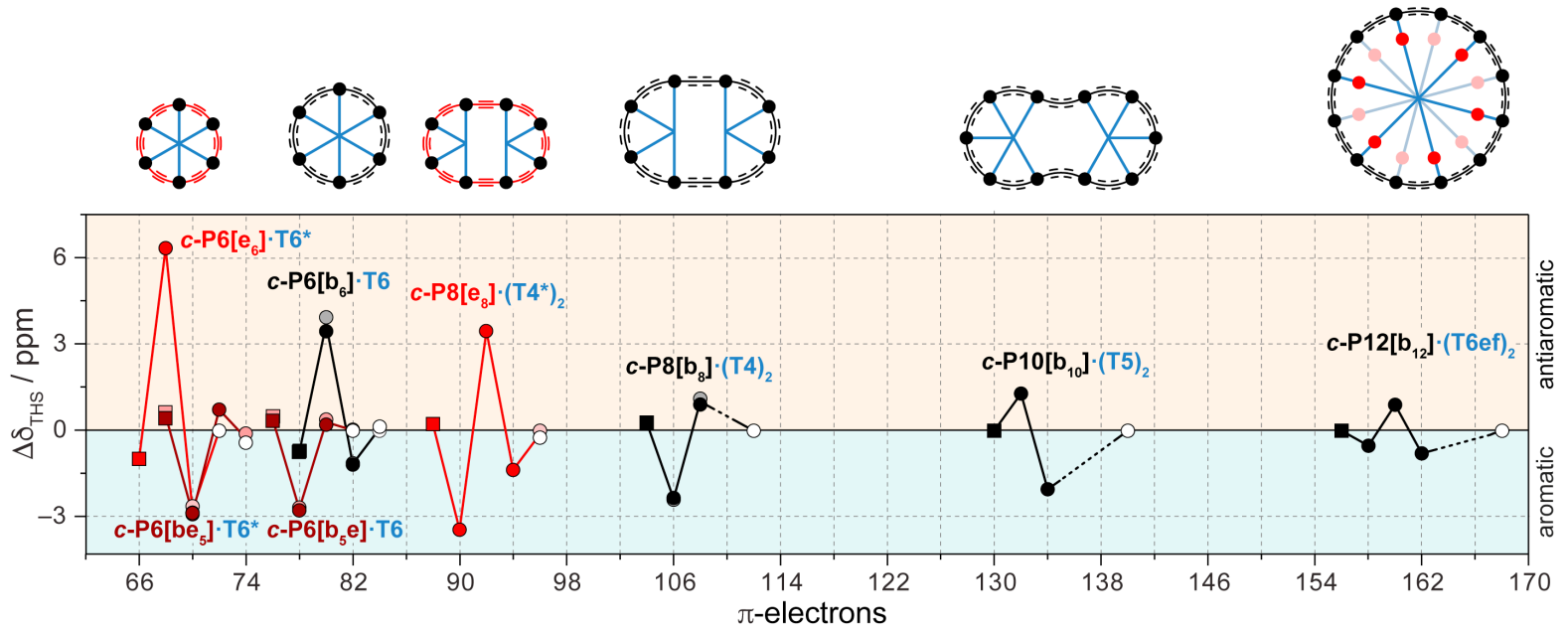

Figure 4. Variation in shielding and deshielding of the THS groups across eight different nanorings.

Top: Schematic representation of a family of related porphyrin rings with increasing number of porphyrins. Red indicates ethyne, black indicates butadiyne linkers. Black dots indicate zinc-porphyrins. Templates are represented in blue. Bottom: Plot of the observed ${ }^{1} \mathrm{H}$ chemical shift difference $\Delta \delta_{\mathrm{THS}}=\delta_{\mathrm{THS}(\mathrm{in})}-\delta_{\mathrm{THS}(\text { out }}$ between inner and outer THS probe for each oxidation state of the nanoring. A positive or negative shift indicates a global antiaromatic or aromatic current, respectively. Empty circles indicate the corresponding neutral species. Squares indicate oxidation states with $\overline{P_{\mathrm{OX}}}=1$. Vertical dashed lines denote oxidation states with $4 n+2 \pi$ electrons. Lines connecting dots are for visual guidance only. $\Delta \delta_{\mathrm{THS}}$ values are shown for both the $\mathrm{CH}_{3}$ signals (dark points) and $\mathrm{SiCH}_{2}$ signals (faded points) of the THS chains.

\section{Methods}

All ${ }^{1} \mathrm{H}$ NMR oxidation titrations were carried out by adding thianthrenium hexafluoroantimonate to a solution of the porphyrin nanoring in $\mathrm{CD}_{2} \mathrm{Cl}_{2}$ at $-60{ }^{\circ} \mathrm{C}$ to -20 ${ }^{\circ} \mathrm{C}$ (see SI for details). At the end of the titration, decamethylferrocene $\left(\mathrm{FeCp}_{2}{ }^{*}\right)$ was added to reduce the porphyrin nanoring back to its neutral form; this process is highly reversible.

\section{Data availability}

The raw NMR spectra and computational data are available from the corresponding author on request. All other data that support these findings are available within the paper or Supplementary Information.

\section{References}

1. Su, T. A., Neupane, M., Steigerwald, M. L., Venkataraman, L. \& Nuckolls, C. Chemical principles of single-molecule electronics. Nat. Rev. 1, 16002 (2016).

2. Gershoni-Poranne, R. \& Stanger, A. Chem. Soc. Rev. 44, 6597-6615 (2015).

3. Chen, Z., Wannere, C. S., Corminboeuf, C., Puchta, R. \& Schleyer, P. v. R. Nucleusindependent chemical shifts (NICS) as an aromaticity criterion, Chem. Rev. 105, 3842-3888 (2005). 
4. Hückel, E. Quantentheoretische Beiträge zum Benzolproblem I. Die Elektronenkonfiguration des Benzols und verwandter Verbindungen. Z. Phys. 70, 204286 (1931).

5. Spitler, E. L., Johnson II, C. A. \& Haley, M. M. Renaissance of annulene chemistry. Chem. Rev. 106, 5344-5386 (2006).

6. Lorke, A. et al. Spectroscopy of nanoscopic semiconductor rings. Phys. Rev. Lett. 84, 2223-2226 (2000).

7. Heckmann, A. \& Lambert, C. Organic mixed-valence compounds: A playground for electrons and holes. Angew. Chem., Int. Ed. 51, 326-392 (2012).

8. Tolbert, L. M. \& Zhao, X. Beyond the cyanine limit: Peierls distortion and symmetry collapse in a polymethine dye. J. Am. Chem. Soc. 119, 3253-3258 (1997).

9. Gieseking, R. L., Ravva, M. K., Coropceanu, V. \& Brédas, J.-L. J. Phys. Chem. C 120, 9975-9984 (2016).

10. Soya, T., Kim, W., Kim, D. \& Osuka, A. Stable [48]-, [50]-, and [52]dodecaphyrins(1.1.0.1.1.0.1.1.0.1.1.0): the largest Hückel aromatic molecules. Chem. Eur. J. 21, 8341-8346 (2015).

11. Yoneda, T., Soya, T., Neya, S. \& Osuka, A. [62]Tetradecaphyrin and its mono- and bis-ZnII complexes. Chem. Eur. J. 22, 14518-14522 (2016).

12. Peeks, M. D., Claridge, T. D. W. \& Anderson, H. L. Aromatic and antiaromatic ring currents in a molecular nanoring. Nature 541, 200-203 (2017).

13. Lu, X. et al. Fluorenyl based macrocyclic polyradicaloids. J. Am. Chem. Soc. 139, 13173-13183 (2017).

14. Cha, W.-Y. et al. Bicyclic Baird-type aromaticity. Nat. Chem. 9, 1243-1248 (2017).

15. Lu, X. et al. Global aromaticity in macrocyclic cyclopenta-fused tetraphenanthrenylene tetraradicaloid and its charged species. Angew. Chem. Int. Ed. 57, 13052-13056 (2018).

16. Gregolińska, H. et al. Fully conjugated [4]chrysaorene. Redox-coupled anion binding in a tetraradicaloid macrocycle. J. Am. Chem. Soc. 140, 14474-14480 (2018).

17. Ke, X.-S. et al. Three-dimensional fully conjugated carbaporphyrin cage. J. Am. Chem. Soc. 140, 16455-16459 (2018).

18. Soya, T., Mori, H. \& Osuka, A. Quadruply twisted Hückel-aromatic dodecaphyrin. Angew. Chem. Int. Ed. 57, 15882-15886 (2018).

19. Li, G. et al. From open-shell singlet diradicaloid to closed-shell global antiaromatic macrocycles. Angew. Chem. Int. Ed. 57, 7166-7170 (2018).

20. Liu, C. et al. Macrocyclic polyradicaloids with unusual super-ring structure and global aromaticity. Chem. 4, 1586-1595 (2018). 
21. Rickhaus, M. et al. Single-acetylene linked porphyrin nanorings. J. Am. Chem. Soc. 139, 16502-16505 (2017).

22. Haver, R. et al. Tuning the circumference of six-porphyrin nanorings, J. Am. Chem. Soc. 141, 7965-7971 (2019).

23. O'Sullivan, M. C. et al. Vernier templating and synthesis of a 12-porphyrin nano-ring, Nature 469, 72-75 (2011).

24. Herges, R. Topology in Chemistry: Designing Möbius molecules, Chem. Rev. 106, 4820-4842 (2006).

25. Stepien, M., Sprutta, N. \& Latos-Grazynski, L. Figure eights, Möbius bands, and more: conformation and aromaticity of porphyrinoids. Angew. Chem. Int. Ed. 50, 4288-4340 (2011).

26. Wirz, L. N., Dimitrova, M., Fliegel, H. \& Sundholm, D. Magnetically induced ringcurrent strengths in Möbius twisted annulenes, J. Phys. Chem. Lett. 9, 1627-1632 (2018).

27. Senthilkumar, K., et al. Lemniscular [16]cycloparaphenylene: A radially conjugated figure-eight aromatic molecule. J. Am. Chem. Soc. 141, 7421-7427 (2019).

28. Schaller, G. R. \& Herges, R. Möbius molecules with twists and writhes. Chem. Commun. 49, 1254-1260 (2013).

29. Kondratuk, D. V., et al. Vernier-templated synthesis, crystal structure, and supramolecular chemistry of a 12-porphyrin nanoring. Chem. Eur. J. 20, 12826-12834 (2014).

30. Edwards, P.P., Lodge, M. T. J., Hensel, F. \& Redmer, R. A metal conducts and a nonmetal doesn't. Phil. Trans. R. Soc. A 368, 941-965 (2010).

Acknowledgments. We thank the EPSRC (grant EP/N017188/1, EP/R029229/1 and EP/M016110/1), the ERC (grant 320969), the European Union's Horizon 2020 research and innovation programme (Marie Sklodowska-Curie grants SYNCHRONICS 643238) and the Swiss National Science Foundation (P300P2_174294) for funding, the National Mass Spectrometry Facility at Swansea University for MALDI spectra and the University of Oxford Advanced Research Computing Service (ARC) for the high performance computing provision (http://dx.doi.org/10.5281/zenodo.22558). MJ thanks Oxford University for a Scatcherd European Scholarship. HG thanks the Carlsberg Foundation for a Carlsberg Foundation Internationalisation Fellowship.

Author contributions. MR, MJ, LT, HG, MDP, RH and H-WJ synthesised the compounds. MR and MJ collected and analysed the NMR spectroscopic data; MJ performed the DFT calculations; TDWC assisted with NMR data collection and interpretation; HLA, MR and MJ devised the project and wrote the paper; all authors discussed the results and edited the manuscript. 